\title{
AMCP Partnership Forum: Building the Foundation for Patient-Reported Outcomes-Infrastructure and Methodologies
}

\begin{abstract}
SUMMARY
Section 3002 of the 21st Century Cures Act, which was signed into law in December 2016, requires the FDA to develop one or more sets of new guidances regarding the collection of patient experience data. To explore how patient-reported outcomes (PROs) can support value-based care and discuss challenges to using PROs more widely, the Academy of Managed Care Pharmacy (AMCP) convened a stakeholder forum in 2017 on PR0s and the role they can play in defining the value of health care interventions. This forum achieved consensus on the importance of PROs in improving patient care through implementing value-based payment models and the need for strong organizational systems to fully adopt and use PROs in health care decision making.

To explore strategies to build on these findings and implement them in practice, AMCP convened a second forum on October 25, 2018, in Orlando, FL, to focus on identifying specific methods and infrastructure to support the ideal use of PROs in practice. In light of the FDA's plans to propose new guidance for incorporating PROs in the regulatory process, this forum focused on the following: (a) describing the current state of using PROs for FDA regulatory and value-based coverage decisions; (b) defining a process for collecting and sharing PROs with the FDA and managed care organizations for value-based decisions; (c) identifying the necessary infrastructure needed to support the ideal use of PR0s; and (d) identifying the health information technology strategies needed to support PRO use across managed care stakeholders.

Strategies for the development and implementation of PROs were discussed throughout the forum, including a focus on appropriate activities to address the needs of patients, health care providers, managed care organizations, and life science companies and researchers. Health care leaders representing health plans, integrated delivery systems, pharmacy benefit managers, employers, national associations of health care professionals, patient advocacy organizations, health infrastructure technology companies and biopharmaceutical companies participated in the forum. The AMCP forum was supported in collaboration with Allergan, Amgen, Boehringer Ingelheim, Bristol-Myers Squibb, Merck, Novo Nordisk, PhRMA, Sanofi, Takeda, and Xcenda.
\end{abstract}

J Manag Care Spec Pharm. 2019;25(4):501-06

Copyright $\odot 2019$, Academy of Managed Care Pharmacy. All rights reserved.

$\mathrm{P}$ atient-reported outcomes (PROs) are a type of clinical outcome assessment that involves the collection of information directly from the patient. This can include self-reported measures of a wide variety of impacts, such as the individual's health status, ability to conduct activities of daily living, pain intensity, and perception of health/treatment. Using PROs to assess a patient's needs and preferences is an important component of the shift toward patient-centered health care. PROs are increasingly being used in assessments of value and cost effectiveness of clinical interventions and endpoints that are included in product labeling. ${ }^{1}$
Federal legal and regulatory developments have helped support these changes. In 2009, the U.S. Food and Drug Administration (FDA) first published a guidance that facilitated inclusion of the patient experience in the drug development process and included PROs as a component of clinical outcome assessments. More recently, Section 3002 of the 21st Century Cures Act of 2016 directed the FDA to issue guidance regarding methods and approaches to be used in capturing and measuring patients' experiences and perspectives. ${ }^{2}$ The FDA aims to address information that is important to patients, as well as patient-generated data (e.g., from wearables and performance tests).

A shift in focus for the FDA is the definition of "patient experience." The 2009 guidance document focused only on measures that are directly related to the disease process. The FDA has more recently shifted the focus to include broader assessments of the burden of disease. For example, measures such as whether people are able to work or participate in social activities are now being more closely considered. Stakeholders are increasingly engaged in practical discussions on how to incorporate the patient voice and perspective throughout drug development, as well as to assign value in value-based care models.

The 2017 Academy of Managed Care Pharmacy (AMCP) stakeholder forum "Improving Quality, Value, and Outcomes with Patient-Reported Outcomes" identified the current use of PROs and opportunities to expand the current use of PROs. ${ }^{1}$ Recommendations that emerged from this forum focused on the need to develop the following:

- A common framework of PRO development, collection, analysis, reporting, and utilization.

- PRO measures that reflect perceptions of relevance to health for patients, clinicians, payers, and regulators.

- Infrastructure and IT systems that support the collection and analysis of PROs.

The AMCP Partnership Forum described here, "Building the Foundation for Patient-Reported Outcomes-Infrastructure and Methodologies," and held October 25, 2018, in Orlando, FL, was convened to gain insights on how to operationalize these recommendations for advancing the use of PROs and to identify recommendations to support PRO use for FDA regulatory decision making and value-based contracting.

\section{Developing and Implementing PROs}

\section{Measure Development}

As PRO collection becomes a component of the systematic evaluation of various interventions, including biopharmaceutical 
products, it is important to have a consistent and defined methodology for developing and implementing PROs.

Participants observed that there are a multitude of factors to consider when developing PROs. Measures must be meaningful to patients and reflect what patients believe about their own health. While metrics already exist for some factors, other domains require development of metrics that provide meaningful measures. Participants noted that caregivers can provide valuable perspectives and could be considered as informants for selected outcomes. Finally, there are initiatives underway to develop core outcome sets for drug development and routine practice use, and some groups have provided item databanks of PROs, such as the Patient-Reported Outcomes Measurement Information System (PROMIS)., 3

\section{PRO Use in Drug Development}

Many prescription drug labels currently include PROs. Participants noted that some of these measures exist because the product treats an outcome that can only be assessed by the patient (e.g., pain). They observed the opportunity to expand the use of PROs in several disease states, including oncology, neurologic disorders, and rare diseases.

Manufacturers are focusing more on "patient-centricity"considering the patient experience-throughout the drug development cycle. Participants noted that researchers are shifting their focus to engage with patients and view them as the experts in the overall experience of their disease. Additionally, manufacturers are increasingly using advisory boards to ensure that the patient perspective is addressed during clinical trial design.

Recommendations for collecting PROs for drug development included identifying disease states and then interviewing relevant stakeholders (e.g., patient advocacy groups) to identify which outcomes are most important and meaningful to patients. For example, disability scores may be of primary importance in the treatment of a condition such as multiple sclerosis, while prevention of disease progression might be most important for patients with type 2 diabetes.

Participants stressed the need to formalize PROs in the drug development process and ensure that these measures are systematically incorporated and addressed. Suggestions included administering PRO measures in phase 2 trials and then assessing the measure performance to fine-tune them for phase 3 trials and developing a central repository of available tools for assessing PROs as a resource for manufacturers. Because some manufacturers may be risk averse, they may prefer to use endpoints that others have used before.

Payer audiences may be interested in phase 4 trials to assess the effect of a therapy on PRO trials to inform their assessments of value. However, phase 4 trials are historically intended to be open-label, long-term safety evaluations of a product and generally are not designed to evaluate PROs.

\section{PRO Use in Value-Based Contracts}

Value-based contracts (VBCs) may be defined as "a written contractual agreement in which the payment terms for medication(s) or other health care technologies are tied to agreed-upon clinical circumstances, patient outcomes, or

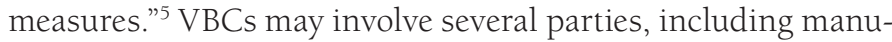
facturers, managed care organizations, providers, and payers.

Participants noted that VBCs typically are based on objective measures. As a result, there are unique challenges for incorporating PROs as outcomes in contracts. Participants focused on the need to assess the validity and appropriateness of a PRO for a VBC. Variables such as disease state, size of the patient population, cost of therapy, and the cost of creating and implementing the PRO would all need to be considered for such an approach. Additionally, participants noted that real-world populations differ from clinical trial populations; therefore, PRO measures used in labeling may not be ideally suited for VBCs.

A key consideration when using PROs in assessments of value for VBCs is how to determine the predictive validity of a PRO as a measure of a meaningful outcome in order to determine how performance on PRO metrics will affect payments. Forum participants suggested linking changes in PRO rating scales to changes in other endpoints, such as hospitalization or resource use, when determining how to assign value. One participant suggested allowing patients to select PROs that they feel are most meaningful and then assessing performance of the intervention based on those individualized PROs. Others noted that this approach would present several challenges for analyzing data, performing comparisons, and determining payments. Goal attainment scaling was suggested as a PRO approach that is designed for patients to identify and track individualized goals. ${ }^{6}$

Because measures of value in VBCs are not always transparent and because standardized, generally accepted metrics are lacking for many PROs, it is difficult to evaluate performance on PROs across contracts. Furthermore, the diversity of measures complicates operations for health care providers, who may be required to assess a domain with several different metrics for different patients depending on a patient's insurance coverage. On the other hand, broad, more widely used measures of quality of life (e.g., the SF-36) many not be sensitive enough to detect changes that are meaningful to patients. Development of standardized disease-specific measures could address some of these challenges.

Participants noted that a patient's life circumstances can dramatically affect measures of health and well-being and explored the effect of social determinants of health on outcomes and PROs. Identifying barriers to care and implementing appropriate supports or connecting patients with community resources can be an important component of improving patient experiences. VBC contracts could be structured so that 


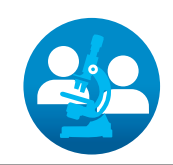

Drug Development Collection Strategies

\begin{tabular}{|c|c|}
\hline Drug Development Collection Strategies & Reporting Strategies \\
\hline \multicolumn{2}{|c|}{ Development } \\
\hline $\begin{array}{l}\text { - Identify stakeholders-patients, relatives, caregivers, clinicians, payers, } \\
\text { and researchers. } \\
\text { - Select/develop measures for outcomes of relevance. } \\
\text { - Qualitative interviews with patients regarding signs and symptoms, } \\
\text { quality of life, function, and preferences. }\end{array}$ & $\begin{array}{l}\text { - Create consensus-based standardized reporting standards that are } \\
\text { measurable, reproducible, and meaningful. } \\
\text { - Validate the financial value of PROs } \\
\text { - Consider how to validate data. }\end{array}$ \\
\hline \multicolumn{2}{|c|}{ Infrastructure } \\
\hline $\begin{array}{l}\text { - Determine where to collect the data (provider office, EHR survey, } \\
\text { personal devices, community pharmacies) and aim to cause the least } \\
\text { disruption to patients. } \\
\text { - Determine who will record the data (patient, physician, caregiver, } \\
\text { pharmacist, nurse, care manager) and consider reporter bias. } \\
\text { - Determine how often to collect data (consider disease state, patient needs, } \\
\text { length of survey). }\end{array}$ & $\begin{array}{l}\text { - Consider the use of registries. } \\
\text { - Develop partnerships with industry and the FDA to address data sharing } \\
\text { across systems and create a platform that can be used by many entities. } \\
\text { - Develop infrastructure for monitoring and interpreting data. }\end{array}$ \\
\hline \multicolumn{2}{|c|}{ Implementation and Use } \\
\hline - Implement PROs in trials in a patient-friendly manner. & - Consider who will own the data. \\
\hline $\begin{array}{l}\text { - Address user interface (dashboard reports, gamification, adaptive designs } \\
\text { for patients with disabilities). }\end{array}$ & $\begin{array}{l}\text { - AMCP could lead collaborations to build models that help define and } \\
\text { promote the value of PROs for contracts. }\end{array}$ \\
\hline $\begin{array}{l}\text { - Educate patients on the use of PRO data at the beginning of a trial so they } \\
\text { understand the importance of reporting. }\end{array}$ & $\begin{array}{l}\text { - Promote clear communication and transparency around reporting } \\
\text { strategies. }\end{array}$ \\
\hline $\begin{array}{l}\text { - Determine who is responsible for collection of data (are there incentives } \\
\text { aligned with data collection?). }\end{array}$ & $\begin{array}{l}\text { - Need to address security of protected health information/deidentification } \\
\text { and aggregation of data. }\end{array}$ \\
\hline $\begin{array}{l}\text { - Provide feedback loops between patients and clinicians that present data } \\
\text { in an actionable format. }\end{array}$ & - Consider opt-in and opt-out strategies for data sharing. \\
\hline - Provide incentives for sustained patient engagement. & \\
\hline
\end{tabular}

payments consider the effect of social determinants of health and encourage strategies to improve patient experiences.

\section{Collecting and Reporting PRO Data}

Assigning responsibility for the collection of PRO data was also considered by participants. For example, should providers receive reimbursement or be accountable for ensuring data collection? Alternatively, should patients be provided with incentives for reporting data? Participants explored opportunities for pharmacists to have a role in PRO data collection and reporting, perhaps during the provision of medication therapy management services, which would also improve patient experience and engagement.

Participants discussed several considerations for collecting and reporting PRO data (Table 1). Several different forms of technology can contribute to effective collection and use of PRO data. Ideally, PRO measures will be integrated seamlessly in electronic health records (EHRs) that are interoperable across providers and systems. However, technology infrastructure limitations will need to be addressed to realize this goal.
Participants identified the need to simplify data collection and develop methodologies that are acceptable for all stakeholders. They recommended avoiding the use of tools that are labor intensive to implement in clinical practice and/or by the patient. They noted that reimbursement is inadequate in exchange for the time required for providers to administer some tools, but also that patient literacy and health literacy must be considered for self-administered tools.

Participants explored the need for innovative strategies to incorporate the collection of PRO data in a manner that is feasible for patients to report information. They suggested the use of newer technologies (e.g., mHealth devices, smartphone apps) to allow for the collection of data but also noted that patient privacy concerns would need to be considered when implementing such approaches. Concerns about the cost of such devices for patients and the effect on patient access would also need to be considered.

Participants highlighted challenges of capturing PRO data accurately within EHR systems and sharing it with other 


\section{TABLE 2 Strategies to Support PRO Use by Managed Care Stakeholders}

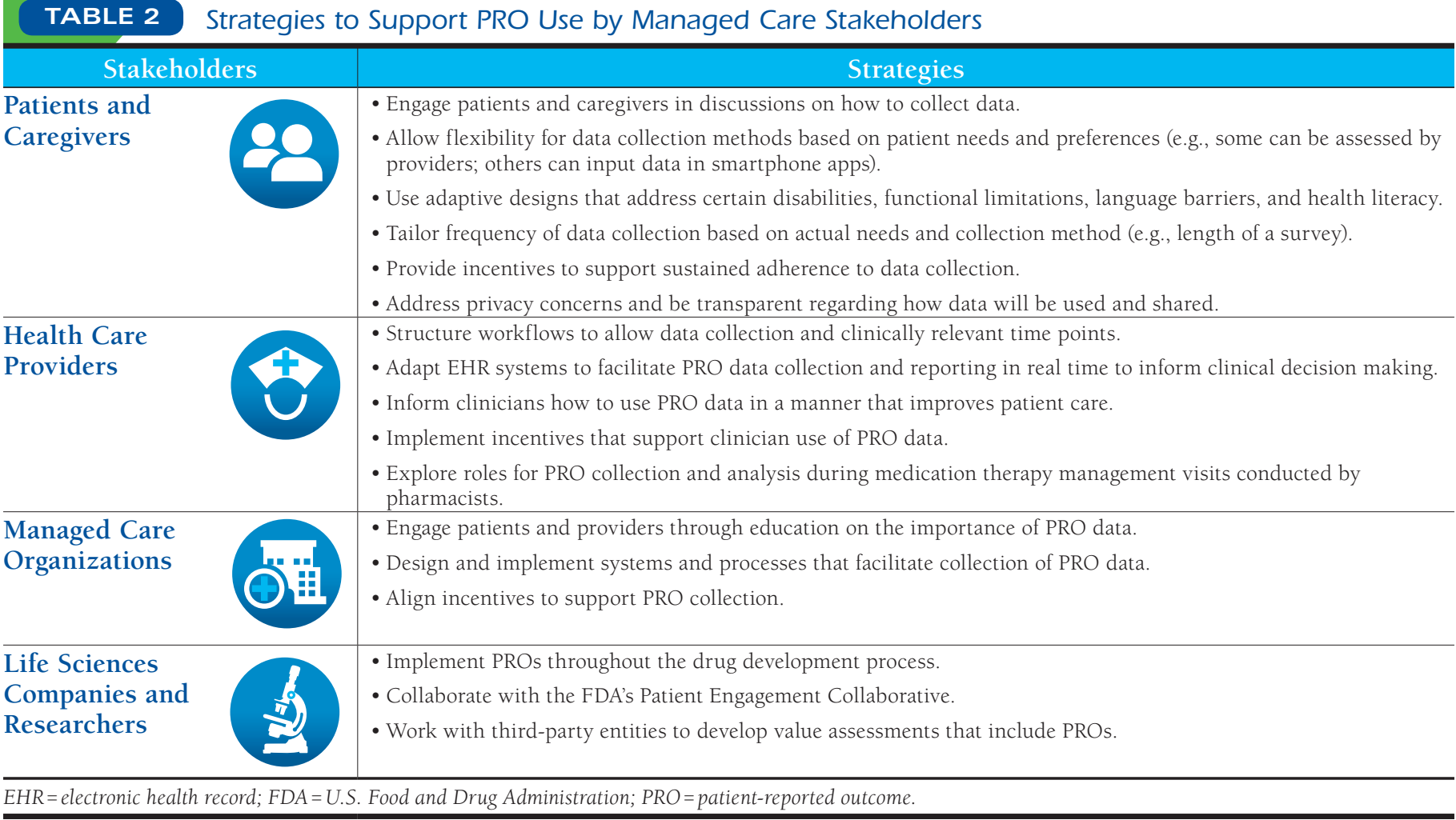

stakeholders. They noted that the collection of data in the real world is less standardized than within clinical trials and that it would be difficult to standardize processes when there is significant variability among measures. Integration of pharmacy and medical data will be needed to effectively and efficiently share PRO data across the health care team.

Participants explored sharing standardized PRO data from EHRs for the administration of VBCs and suggested a centralized source for data, such as a registry. Funding, permissions for accessing data, and processes for deidentification of data would all need to be considered during the development of such a registry. PROs would need to be standardized to allow exchange across interoperable EHR platforms for a registry.

\section{Strategies to Support PRO Use by} Managed Care Stakeholders

Participants shared ideas to support engagement with PROs by several stakeholders, including patients, health care providers, managed care organizations, and life sciences companies and researchers (Table 2). They also addressed challenges and potential solutions.

\section{Patient Engagement in Data Collection}

Because different patient populations have different needs, there must be a multimodal approach to PRO data collection.
For example, some patients may choose an online patient portal to input data; some may require assessments by health care providers in-person or telephonically; and others with access to smartphone apps may prefer that option. Some patients may be willing to frequently engage with reporting, whereas others may prefer less frequent contact. Participants recommended engaging patients and caregivers in discussions regarding strategies for collecting PRO data.

Adaptive designs may be required for patients with certain disabilities or functional limitations. Language barriers and health literacy should be addressed in a way that is feasible, economical, and usable. Gamification-making PRO reporting into a game for the patient-was also discussed as an engagement strategy that could increase adherence to reporting. The use of feedback loops-in which data flows between patients and clinicians in real time and allows data to be actionable and useable-can improve engagement.

The frequency of PRO data collection will be partially driven by the condition being treated, as well as the features of the collection mechanism (e.g., length of a survey). Participants also observed that data collection must be convenient for patients to maintain engagement over time. They suggested the use of incentives to support adherence to PRO data collection over time. They also observed that patients will need to be able to trust the safety of any systems that collect personal information 
and that patients will be more invested in sharing personal data if they feel it is being used to improve their care.

To address privacy concerns that arise with data sharing, participants recommended clear communication and transparency regarding how PRO data will be used. They suggested that AMCP could have a role in facilitating the development of industrywide recommended policies to address PRO data use. They also suggested requiring institutional review board review if data will be used for secondary purposes by other groups.

\section{Health Care Providers}

Forum participants identified factors that can improve the collection of PRO data by health care providers. Structuring work flows to allow data collection at clinically relevant time points is important. Additionally, some EHR systems may need modification to support PRO collection and data sharing. Ideally, PRO data will be available to the patient's health care team in real time and also provide a longitudinal view. Participants also observed that system interoperability is important so that data can be portable if patients change payers and/or care providers.

Participants recommended that pharmacists could be leveraged to facilitate PRO data collection. Pharmacists in many settings are involved in direct patient care and could build questions regarding PROs in their workflow processes and enter data in EHRs. For example, community pharmacists are increasingly involved in medication therapy management and patient care services; specialty pharmacists are very involved with patient management and conducting disease activity assessments; and health-system pharmacists are most likely to already have access to EHR systems that are shared by teams.

There are barriers to the use and actionability of PRO data. Participants emphasized the need to examine provider workflow and implement incentives that support clinician use of the data. There is also a need to build awareness around how to use PRO data in a meaningful manner to improve patient care. EHR systems should be developed around gathering the appropriate data and ensuring that it is available to appropriate individuals to support informed decision making.

\section{Managed Care Organizations}

Participants saw an important role for managed care organizations in engaging patients and providers and educating them about the importance of PRO data collection. They also felt that managed care organizations could help facilitate data collection and assist with designing and implementing systems and processes that allow collection to occur in a manner that is convenient for patients, such as through the creation of multiple platforms (e.g., through an EHR portal and a smartphone app). They also stressed that managed care organizations should align incentives with data reporting and provide patients with positive feedback when they report data. They noted that the creation of quality metrics based on PROs would be informative and could stimulate data collection.

\section{Life Sciences Companies and Researchers}

The FDA has established pathways for manufacturers and researchers to integrate PROs into the drug development process. For example, manufacturers can prioritize the inclusion of PROs in the primary or secondary outcomes of clinical trials and request a joint meeting with the Patient Engagement Collaborative at the FDA during the drug approval process. These strategies can help ensure that appropriate experts evaluate the relevant clinical and patient-reported data that could potentially be used in an approved drug label. ${ }^{7}$ Participants also suggested working with third-party entities such as the Institute for Clinical and Economic Review or ISPOR-The Professional Society for Health Economics and Outcomes Research to develop value assessments that include PROs.

Additional financial support may be needed for the collection of PROs outside of a clinical trial setting, since adequate financial supports may be available during clinical trials but typically are lacking in other settings.

\section{Creating a Plan to Promote Implementation of PROs}

Participants provided several suggestions for supporting the implementation of PROs in drug development and VBCs and envisioned several potential roles for AMCP to facilitate these activities.

AMCP could play an important role in working with pharmacies and EHR vendors to incorporate PROs into workflows so that data can be collected, evaluated, and used for clinical decision making at the point of care. Additionally, participants recommended that AMCP continue to foster collaborations and convene meetings among various stakeholders and organizations. A PRO workgroup could be created and tasked with prioritizing issues and developing standards. Organizing and convening "connect-a-thons" to facilitate the development of tools for PRO collection, foster collaboration, and develop solutions to barriers was proposed as another strategy. AMCP could also serve as a centralized source for tools and resources that are developed for PRO implementation and use these materials in education and advocacy efforts.

Recommended education and advocacy efforts included the creation of position papers to promote awareness about the value of PROs. Participants also recommended that AMCP create and validate models for PROs use and work with various entities (e.g., Centers for Medicare \& Medicaid Services, National Institutes of Health, and the Patient-Centered Outcomes Research Institute) to address questions regarding the use of PROs outside the FDA regulatory environment. 


\section{Conclusions}

PROs are increasingly being valued during development and approval processes for biopharmaceutical products. When developing metrics for PROs, it is important to carefully balance the need for simplicity and standardization with the need to have sensitive and clinically relevant measures for various disease states. Ultimately, PROs should be designed to detect changes that provide value that is meaningful to patients.

In the postapproval environment, standardizations for PROs can help create a framework to inform best practices around care delivery and coverage determinations. Manufacturers are interested in having PROs incorporated in clinical trial design to help support drug approval and meaningful comparisons across drugs and coverage determinations and as a basis for FDA approval.

Improved health information technology infrastructure, including interoperability of EHR systems, will be necessary to support effective use of PROs across the health care system.

Finally, participants recommended facilitating cross-industry partnerships and public-private partnerships to facilitate the development and implementation of strategies to enhance implementation.

\section{Forum Participants}

AMANDA BAIN, Director, Pharmacy and Care Management, The Ohio State University Health Plan; KRISTIN BOROWSKI, Senior Manager, Federal Payment Agencies, Quality, Bristol-Myers Squibb; JOEL BRILL, Chief Medical Officer, Predictive Health; ERIC CANNON, Vice President, Pharmacy Benefits, SelectHealth; NIKKI CARRICO, Pharmacist, Epic; ALANA CLEMENSSALIBA, Associate Director, Patient Advocacy and Professional Relations, Boehringer Ingelheim; JOSEPH COUTO, Data Science Director, Cigna Healthcare; GWEN DARIEN, Executive Vice President, Patient Advocacy, Patient Advocate Foundation and National Patient Advocate Foundation; JESSICA DAW, Senior Director, Clinical Pharmacy, UPMC Health Plan; MARTINE ETSCHMAIER, Associate Director, Xcenda; LISA GAUVIN, Pharmacy Manager, Aetna Specialty Pharmacy; DIANA GRAALUM, Clinical Pharmacy Manager, MedSavvy; MARK GREGORY, Director, Pharmacist Consultant, Med Adherence Division, Omnicell; CAROLYN HA, Director, Policy \& Research, PhRMA; JONATHAN HAMRICK, President and Chief Operating Officer, Therigy; KATHERINE HARRIGAN, HEOR Fellow, Takeda; PATRICIA JACOB, Director, Managed Care Scientific, Allergan; MARK KOSINSKI, Vice President, Life Sciences and Patient Insight/Senior Scientist, QualityMetric/Optum; GREG LOW, Director, MGPO Pharmacy Quality \& Utilization Program, Massachusetts General Hospital; DANA MCCORMICK, Director of Pharmacy, Blue Cross Blue Shield of Texas; TRENT MCLAUGHLIN, Vice President, Real World Evidence, Xcenda; MICHAEL NEUFELD, Senior Associate Director, Payor Solutions, Boehringer Ingelheim Pharmaceuticals; LISA OPIPARI, Associate Professor of Pediatrics, Cincinnati Children's Hospital Medical Center;
LESLIE RITTER, Senior Director, Federal Government Relations, National Multiple Sclerosis Society; CYNTHIYA RUBAN, Analyst, GHEOR, Xcenda; KENNETH SCHAECHER, Associate Chief Medical Officer, University of Utah Health Plans; STEVEN SMITH, Health Systems Medical Affairs Director, Merck; TRACY SPINKS, Senior Director, Quality Innovation, National Quality Forum; KIMBERLY STERLING, Director, Health Economics and Value Assessment, Sanofi, MEREDITH STROZIER, Director, Practice Advocacy, American College of Rheumatology; MICHAEL SULLIVAN, US Value and Access Portfolio Marketing Director, Amgen; PENNY SURRATT, Senior Director, Trade Relations, ReCept Healthcare Services; SHEILA M. THOMAS, Head, Patient Insights \& Engagement Strategy, Sanofi; ROBIN TURPIN, Value Evidence and Health Outcomes Scientific Lead, Takeda; MARGARET VERNON, Vice President, Patient-Centered Research, Evidera; and WENDY WEINGART, Senior Vice President, Managed Care Services, Visante.

CORRESPONDENCE: Tricia Lee Wilkins, Academy of Managed Care Pharmacy, 675 N. Washington St., Ste. 220, Alexandria, VA 22314. Tel.: 703.684.2641; E-mail: tlwilkins@amcp.org.

\section{DISCLOSURES}

The AMCP Partnership Forum and the development of the proceedings document were supported by Allergan, Amgen, Boehringer Ingelheim, BristolMyers Squibb, Merck, Novo Nordisk, PhRMA, Sanofi, Takeda, and Xcenda.

\section{ACKNOWLEDGMENTS}

The AMCP Partnership Forum "Building the Foundation for PatientReported Outcomes: Infrastructure and Methodologies" was moderated by Caleb Alexander, Co-director, Johns Hopkins Center for Drug Safety and Effectiveness, Johns Hopkins Bloomberg School of Public Health. These proceedings were written by Judy Crespi Lofton, Medical Writer and Consultant, JCL Communications.

\section{REFERENCES}

1. AMCP Partnership Forum: Improving quality, value and outcomes with patient-reported outcomes. J Manag Care Spec Pharm. 2018;24(3):304-10. Available at: https://www.jmcp.org/doi/10.18553/jmcp.2018.17491.

2. U.S. Food and Drug Administration. Plan for issuance of patient-focused drug development guidance under 21 st Century Cures Act Title III Section 3002. May 2017. Available at: https://www.fda.gov/downloads/forindustry/userfees/prescriptiondruguserfee/ucm563618.pdf. Accessed February 26, 2019.

3. National Institutes of Health. FDA standard core clinical outcome assessments and endpoints. Notice Number: NOT-FD-18-014. July 31, 2018. Available at: https://grants.nih.gov/grants/guide/notice-files/NOTFD-18-014.html. Accessed February 26, 2019.

4. National Institutes of Health. Patient-Reported Outcomes Measurement Information System (PROMIS) program snapshot. Available at: https://commonfund.nih.gov/promis/index. Accessed February 26, 2019.

5. AMCP Partnership Forum: Advancing Value-Based Contracting. J Manag Care Spec Pharm. 2017;23(11):1096-102. Available at: https://www.jmcp.org/ doi/10.18553/jmcp.2017.17342

6. Krasny-Pacini A, Hiebel J, Pauly F, et al. Goal attainment scaling in rehabilitation: a literature-based update. Ann Phys Rehabil Med. 2013;56(3):212-30.

7. U.S. Food and Drug Administration. Learn about FDA patient engagement. Available at: https://www.fda.gov/forpatients/patientengagement/ default.htm. Accessed February 26, 2019. 